\title{
Complete dysphagia after thrombolytic treatment for myocardial infarction
}

\author{
T Mosimann, L Terracciano, C Sieber, F Rappo
}

\begin{abstract}
An 82 year old man was admitted to hospital with unstable angina pectoris. There was a long history of minor symptoms suggesting reflux disease, with a small diaphragmatic hernia. One day after admission the patient complained of severe chest pain. An acute inferiorposterior myocardial infarction was diagnosed on ECG, and thrombolytic treatment with alteplase (rt-PA) was initiated. Within a few hours total dysphagia occurred, caused by haemorrhagic oesophagitis. The haematoma resolved spontaneously within about 10 days. The patient was discharged three weeks later after full resolution of the dysphagia. (Postgrad Med F 2000;76:795-796)
\end{abstract}

Keywords: haemorrhagic oesophagitis; thrombolytic treatment; bleeding; myocardial infarction

Although gastro-oesophageal reflux disease with subsequent oesophagitis is very common, and thrombolytic treatment for myocardial infarction is a routine treatment, there has been only one case report of haemorrhagic oesophagitis with complete dysphagia after thrombolysis. ${ }^{1}$ Given the high incidence of gastrooesophageal reflux, it is possible that lesser degrees of haemorrhagic oesophagitis may occur in this situation more often than has been recognised.

Internal Medicine,

Thierstein Hospital, 4042 Breitenbach, Switzerland

T Mosimann

F Rappo

Department of Pathology, University Hospital Basle, 4031

Basle, Switzerland

L Terracciano

Department of Gastroenterology, University Hospital Basle

C Sieber

Correspondence to: Dr T Mosimann, Department of Internal Medicine, Kantonsspital Luzern, 6000 Luzern 16, Switzerland

thierry.mosimann@

datacomm.ch

Submitted 26 October 1999 Accepted 10 February 2000

\section{Case report}

An 82 year old patient was admitted to hospital with a diagnosis of unstable angina pectoris. $\mathrm{He}$ was being treated with aspirin $100 \mathrm{mg}$ daily and there was no history of other non-steroidal anti-inflammatory drugs. There was also a history of infrequent dyspeptic symptoms.

On the next day the patient complained of sudden severe chest pain. The tentative diagnosis of an acute inferior-posterior myocardial infarction was confirmed by ECG. Thrombolytic treatment with alteplase (rt-PA) was initiated. A few hours later, the patient developed odynophagia, severe chest pain radiating into the right shoulder, and complete dysphagia. On day 5, parenteral feeding had to be started.

On day 7, an oesophagogastroduodenoscopy was performed. At $18 \mathrm{~cm}$ from incisors, the lumen was nearly blocked by necrotic debris and bleeding tissue, and $30 \mathrm{~cm}$ from incisors the lumen was totally obstructed. These findings suggested an oesophageal carcinoma.

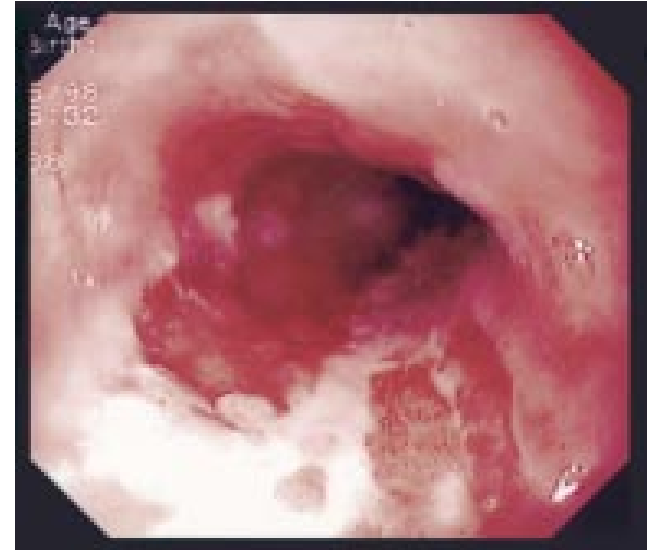

Figure 1 Endoscopy performed before discharge showing grade 4 oesophagitis with ulceration and fibrin clots.

The differential diagnosis included exfoliative inflammation. Over the next 10 days the dysphagia improved steadily. Eleven days after the onset of the symptoms the patient was able to swallow a soft diet. The endoscopic biopsies showed typical severe oesophagitis with no malignant or dysplastic cells. Endoscopy performed before discharge showed grade 4 oesophagitis (fig 1). Seventeen days after the thrombolytic treatment the patient could eat a normal diet. He patient was discharged in good general condition after three weeks.

\section{Discussion}

Thrombolysis in acute myocardial infarction is a well established treatment. The complication of severe haemorrhage is a rare but also much dreaded event, and occurs in around $1-4 \%$ of all the patients undergoing thrombolytic treatment. ${ }^{2}{ }^{3}$ However, the total number of haemorrhagic complications (including small bleeds) may be as high as $30 \%$. $^{3}$ Around $6 \%$ of the bleeding complications occur in the gastrointestinal tract. ${ }^{3}$ The three monthly prevalence of subjectively relevant dyspeptic complaints in Switzerland in 1997 was about $25 \%{ }^{4}$ According to published reports, oesophagitis can be endoscopically confirmed in $48-79 \%$ of this group of patients. ${ }^{5}$ Overall, the prevalence of endoscopically proven oesophagitis is around $10 \%$ in Switzerland, and around $5 \%$ in the international literature. ${ }^{5}$ If the prevalence of oesophagitis in patients with acute coronary thrombosis is not different from that of the population as a whole, one would expect to find potentially haemorrhagic reflux oesophagitis in every 10th patient in Switzerland. However, the patient's case history does not usually help 


\section{Summary points}

- Haemorrhages in the gastrointestinal tract account for $6.4 \%$ of all bleeding complications of thrombolytic treatment.

- The prevalence of endoscopically proven oesophagitis is about $10 \%$ in Switzerland. In the international literature, this figure is around 5\%.

- Haemorrhagic oesophagitis can lead to total dysphagia.

- The incidence of dysphagia caused by haemorrhagic oesophagitis following thrombolytic treatment is probably much higher than previously reported.

the physician to assess the individual risk of haemorrhage resulting from gastrooesophageal reflux disease. It is generally accepted that the intensity of reflux symptoms does not correlate with the endoscopic findings. On the contrary, it has been found that endoscopically proven ulcerative oesophagitis tends to cause few or no complaints. Given the figures mentioned above, one would expect a higher incidence of haemorrhagic oesophagitis following thrombolytic treatment than appears to be the case. However, milder forms of oesophageal haemorrhage without obvious dysphagia have been reported quite often. ${ }^{5}$
Our patient had oesophagogastroduodenoscopy six and 10 days after myocardial infarction. Given the serious and sudden onset symptoms of odynophagia and total dysphagia, endoscopy was required in this case, and the patient was considered medically stable. However, this procedure should be performed only while monitoring vital signs, and endotracheal intubation and mechanically assisted ventilation should be available. ${ }^{5}$

To the best of our knowledge this is only the second case where haemorrhagic reflux oesophagitis and total dysphagia developed so dramatically within a few hours after initiation of thrombolytic treatment. ${ }^{1}$

1 Jishi F, Sissons CE, Silverstone EJ, et al. Oesophageal dissection after thromolytic treatment for myocardial infarction. Thorax 1992;47:835-6.

2 Berkowitz SD, Granger CB, Pieper KS, et al. Incidence and predictors of bleeding after contemporary thrombolytic therapy for myocardial infarction. Circulation 1997;95: 2508-16.

3 McLeod DC, Coln WG, Thayer CF, et al. Pharmacoepidemiology of bleeding events after use of r-alteplase or streptokinase in acute myocardial infarction. Am Pharmacostreptokinase in acute $1993 ; 27: 956-62$.

4 Giger M, Brignoli R. Prävalenz von Oberbauchschmerzen und deren Einfluss auf Lebensqualität und Konsum von medizinischen Leistungen. Schweiz Med Wochenschr 1998; 128:874-9.

5 Cappell MS. The safety and clinical utility of oesophagogastroduodenoscopy for acute gastrointestinal bleeding after myocardial infarction: a six-year study of 42 endoscopies in 34 consecutive patients at two university teaching hospitals. Am f Gastroenterol 1993;88:344-50. 